\title{
Simultaneous improvement of tensile strength and ductility in micro-duplex structure consisting of austenite and ferrite
}

\author{
L. Chen, F.P. Yuan, P. Jiang, J.J. Xie, X.L. Wu* \\ State Key Laboratory of Nonlinear Mechanics, Institute of Mechanics, Chinese Academy of Science, Beijing 100190, PR China
}

\section{A R T I C L E I N F O}

\section{Article history:}

Received 16 April 2014

Received in revised form

5 September 2014

Accepted 6 September 2014

Available online 16 September 2014

Keywords:

Duplex stainless steel

Equal channel angular pressing

Strain hardening

Modeling

Phase boundary

\begin{abstract}
A B S T R A C T
A micro-duplex structure consisting of austenite and ferrite was produced by equal channel angular pressing and subsequent intercritical annealing. As compared to coarse-grained (CG) counterpart, the strength and ductility of micro-duplex samples are enhanced simultaneously due to smaller grain sizes in both phases and more uniformly distributed austenite in ferrite matrix. The average yield stress and uniform elongation are increased to $540 \mathrm{MPa}$ and 0.3 as compared to $403 \mathrm{MPa}$ and 0.26 of its CG counterpart respectively. The Hall-Petch coefficients of austenite and ferrite grain boundaries were quantitatively measured as 224.9 and $428.9 \mathrm{MPa} \mu \mathrm{m}^{1 / 2}$ respectively. In addition, a Hall-Petch type coefficient was used to describe the ability of phase boundary to obstruct dislocation motion, which was measured as $309.7 \mathrm{MPa} \mu \mathrm{m}^{1 / 2}$. Furthermore, the surface-to-volume ratio of phase boundary in microduplex structure was estimated to be $1.17 \times 10^{6} \mathrm{~m}^{-1}$, which is increased by an order of magnitude as compared to $1.2 \times 10^{5} \mathrm{~m}^{-1}$ of its CG counterpart. Based on the strain gradient theory, a model was proposed to describe the effect of surface-to-volume ratio of phase boundary on strain hardening rate, which shows a good agreement with the experimental results.
\end{abstract}

(c) 2014 Elsevier B.V. All rights reserved.

\section{Introduction}

Extensive investigations over the past few decades have demonstrated that the nanostructured (NS) materials have poor tensile ductility although the strength is significantly increased as compared to their CG counterparts [1-4]. The low tensile ductility of NS materials is mainly attributed to their low strain hardening ability because the conventional dislocation mechanism is suppressed by the extremely small grains $[1,5]$. However, some researches during past decade also exhibit that the well-designed microstructures could achieve high strain hardening ability, including the introduction of a gradient or bimodal grain size distribution [6,7], the preexisting nano-scale growth twins [2], dispersion of nano-precipitates [8,9], transformation and twinning induced plasticity [10,11], and a mixture of two or multiple phases with varying size scales and properties [12].

Many natural and man-made materials consist of dual or multiple phases, which make them exhibit much better strengthductility synergy than those single phase materials [13]. To elucidate the relationship between microscopic mechanical behaviors and bulk mechanical properties of dual and even multiple phase materials, more and more advanced in-situ experiments and

\footnotetext{
* Corresponding author.

E-mail address: xlwu@imech.ac.cn (X.L. Wu).
}

computer simulations were conducted over the past two decades [14-18]. In terms of micro-mechanics, three conclusions could be addressed from those investigations. Firstly, the soft phase always tends to yield earlier than the hard one, leading to an inhomogeneous distribution of plastic strain across phase boundary even under uniaxial tensile test. Thus, secondly, the plastic strain gradient occurs between two dissimilar phases when plastic deformation happens. Thirdly, with different plastic strain accommodated by the hard and soft phases, the applied load born by hard phase is greater than that by the soft, resulting in inhomogeneous stress partitioning between two phases.

The above plastic deformation features are believed to be responsible for the optimized mechanical properties of dual and multiple phase materials. During plastic deformation, the hard phase is relatively elastically deformed and bears most of the applied load, while the soft one provides strain hardening ability and accommodates most of the plastic deformation. Thus high strength and good tensile ductility could be achieved simultaneously. Moreover, the plastic strain gradient across phase boundary requires the generation of geometrically necessary dislocations (GNDs), which would lead to an extra strength over the rule-of-mixture (ROM) prediction. In addition, with grain size decreasing to nanometer range, the lattice dislocations could glide on the phase boundaries and penetrate them into adjacent phases [19-22]. The phase boundary therefore play a similar role for NS dual phase materials as the twin boundary does for 
nano-twinned metals, thus reducing the strain localization and enhancing the interaction between dislocations during plastic deformation [2].

Since the interaction between two phases results from the strength difference in essence, it is reasonable to deduce that those features of inhomogeneous stress and strain distribution also occur in NS dual phase materials if strength difference still exists. This interaction has a potential to improve the dislocation storage ability due to the generation of GNDs, especially considering the increased surface-to-volume ratio of phase boundary. In the case of $\mathrm{Cu} / \mathrm{M}(\mathrm{M}=\mathrm{Nb}, \mathrm{Ta}, \mathrm{Fe}$, etc.) $\mathrm{NS}$ composites, the bulk strengths tested by experiments exceed the ROM predictions [23]. To describe this strengthening behavior qualitatively, an additional term of yield stress was introduced by considering the interplay between the phases $[24,25]$.

Although promising in mechanical properties [26-30], the contributions of individual phase to the overall strength and strain hardening are difficult to analyze quantitatively, which are important in establishing the microscopic mechanical models and tailoring the macroscopic mechanical properties. These naturally raise two fundamental questions: How strong does the phase boundary impede the dislocation motion as compared to the grain boundaries of individual phases? And how much contribution does the phase boundary have to the overall strain hardening ability of duplex microstructure?

In the current study, the influences of phase boundary on strength and strain hardening rate are investigated. The microduplex samples were fabricated by equal channel angular pressing (ECAP) and subsequent intercritical annealing. Then the strengthening abilities of grain and phase boundaries were quantitatively analyzed. Moreover, the strain gradient theory was used to describe the effect of phase boundary on strain hardening behavior of micro-duplex structure.

\section{Experimental procedures}

The UNS S32304 duplex stainless steel (DSS) was used in this investigation, with chemical compositions (wt\%) of $0.02 \mathrm{C}$, $0.5 \mathrm{Si}, 1.2 \mathrm{Mn}, 23.5 \mathrm{Cr}, 4.0 \mathrm{Ni}, 0.4 \mathrm{Mo}, 0.13 \mathrm{~N}, 0.024 \mathrm{P}, 0.002 \mathrm{~S}$, and balanced Fe.

The as-received billets of $10 \mathrm{~mm}$ in diameter were annealed at $1373 \mathrm{~K}$ for $2 \mathrm{~h}$, followed by oil quenching in vacuum of about $10^{-4} \mathrm{~Pa}$, in order to obtain the CG samples with nearly 50:50 phase balance between austenite and ferrite.

To fabricate the micro-duplex samples, the as-received billets of $10 \mathrm{~mm}$ in diameter were firstly solutionized at $1623 \mathrm{~K}$ for $2 \mathrm{~h}$ to form single ferrite microstructure (in vacuum of about $10^{-4} \mathrm{~Pa}$ and followed by oil quenching). ECAP technique was then used to refine the grain size of ferrite via a split die with two channels intersecting at inner angle of $90^{\circ}$ and outer angle of $30^{\circ}[31,32]$.
The ECAP was conducted at ambient temperature for 1 pass since further pressing is hugely difficult. At last, the ECAPed samples were intercritically annealed at $1173 \mathrm{~K}$ for different time to generate micro-duplex structure (in vacuum of about $10^{-4} \mathrm{~Pa}$ and followed by water quenching).

The dog-bone shaped tensile specimens were designed with rectangular cross-section of $2 \times 1 \mathrm{~mm}^{2}$ and gauge length of $8 \mathrm{~mm}$ and machined by electrical discharging along extrusion direction on the $Y$ plane [32,33]. Tensile tests were conducted using an Instron 8871 test machine at room temperature with strain rate of $5 \times 10^{-4} \mathrm{~s}^{-1}$. At least three times of tensile testing were conducted for each microstructure.

An Olympus PMG3 optical microscope (OM) was used to examine the microstructures and measure the phase fractions. The chemical etchant used for OM observation consists of $30 \mathrm{~g}$ $\mathrm{K}_{3} \mathrm{Fe}(\mathrm{CN})_{6}, 10 \mathrm{~g} \mathrm{KOH}$ and $100 \mathrm{ml}_{2} \mathrm{O}$. Remaining the solution temperature at $353 \mathrm{~K}$, the OM sample was immersed into it for 3 min.

X-ray diffraction (XRD) was taken to investigate the effect of tensile deformation on volume fraction of individual phases by using Rigaku D/max 2400 X-ray diffractometer with $\mathrm{Cu} \mathrm{K}_{\alpha}$ radiation, and a step size of $0.02^{\circ}$.

The micro-duplex structure before and after tensile tests was investigated by electron back-scattered diffraction (EBSD) using a field emission gun scanning electron microscope. Specimens for EBSD investigation were prepared on the $Y$ plane by standard mechanical grinding and polishing procedures. In the final step, samples were electro-polished using a solution of 95\% ethyl alcohol and $5 \%$ perchloric acid $\left(\mathrm{HClO}_{4}\right)$ at $253 \mathrm{~K}$ with voltage of $38 \mathrm{~V}$. The EBSD scans were carried out at $15 \mathrm{kV}$ in the center of the gauge section at a step size of $100 \mathrm{~nm}$. The raw data were postanalyzed using TSL OIM software, and the average misorientation of a given point relative to its neighbors was calculated using an orientation gradient kernel method. In this study, the kernel average misorientation (KAM) was calculated up to the second neighbor shell with a maximum misorientation angle of $2^{\circ}$.

\section{Results}

\subsection{Mechanical property}

Uniaxial tensile tests were conducted to investigate the mechanical properties of different dual phase microstructures. The engineering stress-strain curves are shown in Fig. 1(a). The yield stress of micro-duplex samples annealed for $10 \mathrm{~min}$ at $1173 \mathrm{~K}$ is increased to $540 \mathrm{MPa}$, as compared to $403 \mathrm{MPa}$ of CG DSS. More importantly, the uniform elongation is increased simultaneously, which is 0.3 as compared to 0.26 of CG DSS.
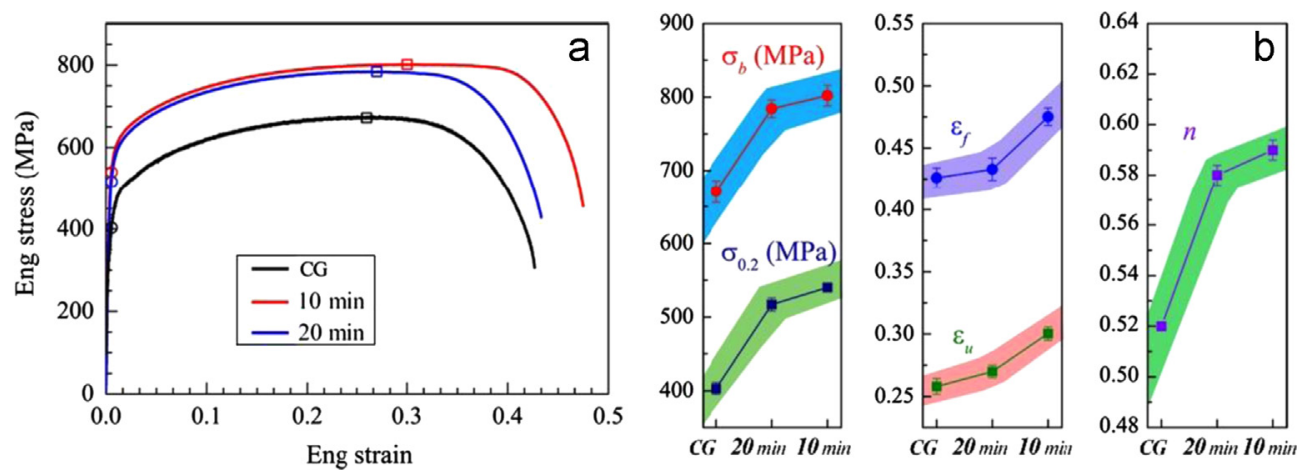

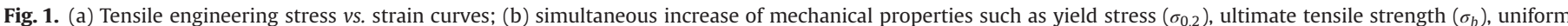
elongation $\left(\varepsilon_{u}\right)$, elongation to failure $\left(\varepsilon_{f}\right)$ and strain hardening exponent $(n)$. 
The corresponding mechanical properties, including the yield stress, ultimate tensile stress, uniform elongation, elongation to failure and strain hardening exponent, are shown in Fig. 1(b). The strain hardening exponents were calculated using the Ludwik equation [34]. The micro-duplex sample annealed for $20 \mathrm{~min}$ appear small weak mechanical properties as compared to that annealed for $10 \mathrm{~min}$, manifesting better mechanical performance for finer-grained dual phase microstructure.

\subsection{Microstructure observation}

The mechanical properties of DSS depend closely on the volume fraction and phase morphology of austenite, as well as the grain sizes of austenite and ferrite. The dual phase microstructure could be adjusted effectively by the combination of plastic deformation and intercritical annealing. For example, at relatively high annealing temperature, the deformed nonequilibrium ferrite would recrystallize first and followed by precipitation of austenite along grain boundaries of ferrite. But when annealed at relatively low temperature, the austenite would precipitate first from deformed ferrite along its (sub-) grain boundaries, and therefore suppress the recrystallization of ferrite. These two different thermodynamic processes lead to different dual phase microstructures, especially in phase morphology and volume fraction of austenite [35]. Therefore, the ECAPed samples with single ferrite phase were annealed at different temperatures to obtain a dual phase microstructure with finer grains and more uniform morphology to achieve better mechanical properties.

Fig. 2(a) shows the microstructure of CG DSS, in which the austenite islands embedded in ferrite matrix are about $20 \mu \mathrm{m}$ in transversal size. The ECAPed samples were intercritically annealed at $1173 \mathrm{~K}$ and $1273 \mathrm{~K}$ for same $30 \mathrm{~min}$. Fig. 2(b) shows the microstructure annealed at $1173 \mathrm{~K}$, in which the austenite islands precipitate along the previous shear flow lines produced by ECAP. When annealed at $1273 \mathrm{~K}$, as shown in Fig. 2(c), nearly equiaxed austenite islands are exhibited. Based on Fig. 2(b) and (c), finer austenite and ferrite could be obtained if annealed at $1173 \mathrm{~K}$. Because at this temperature, the austenite precipitate first and suppress the recrystallization of ferrite, retaining the small size of ferrite. Fig. 2(d) shows the transversal size distributions of austenite and ferrite exhibited in Fig. 2(b), and both the average sizes are typically less than $2 \mu \mathrm{m}$.

The micro-duplex structure depends on not only the intercritical annealing temperature, but also the annealing time. Keeping the temperature at $1173 \mathrm{~K}$, two groups of ECAPed samples were intercritically annealed for 10 and 20 min. Fig. 3(a) displays the micrograph of image quality (IQ) overlapped with inverse pole figure (IPF) for the specimen annealed for $10 \mathrm{~min}$. The volume fraction of austenite is $48 \%$. Corresponding to Fig. 3(a), (b) and (c) are the IPF and grain boundary distribution maps of austenite and ferrite respectively. Fig. 3(d) shows the IQ micrograph overlapped with phase map for the sample annealed for $20 \mathrm{~min}$. The volume fraction of austenite is $49 \%$. Fig. 3(e) and (f) is the corresponding IPF with grain boundary distribution maps of austenite and ferrite in Fig. 3(d). Based on the comparison of two sets of micrographs, it could be concluded that both the austenite and ferrite are coarsened with annealing time increasing. Therefore, a short time annealing is beneficial to keep fine-grained dual phase microstructure.

The statistical distributions of grain sizes of austenite and ferrite in Fig. 3(a) and (d) are shown in Fig. 4(a). The grain diameters are determined by assuming spherical grains in shape for both phases. The average grain size of austenite is smaller than that of ferrite in both annealing conditions. It can be seen in Fig. 4(a) that grain size of ferrite is far greater than that of austenite when annealed for $20 \mathrm{~min}$, which may be caused by the coalescence of ferrite. For comparison, the corresponding distributions of intercept length (with 69 horizontal lines) in two phases are depicted in Fig. 4(b). The average intercept lengths of austenite grains are also smaller than that of ferrite grains in both conditions.

Austenite is always metastable and could transform to martensite during tensile deformation. But in DSS used in this investigation, austenite is very stable and there is no detectable martensite transformation upon tensile test. Fig. 5 shows the XRD spectra of two micro-duplex samples before and after tensile deformation. The relative intensity of all the main characteristic peaks is nearly not changed with tensile strain. It manifests that the austenite could keep stable during tensile deformation. Our previous research
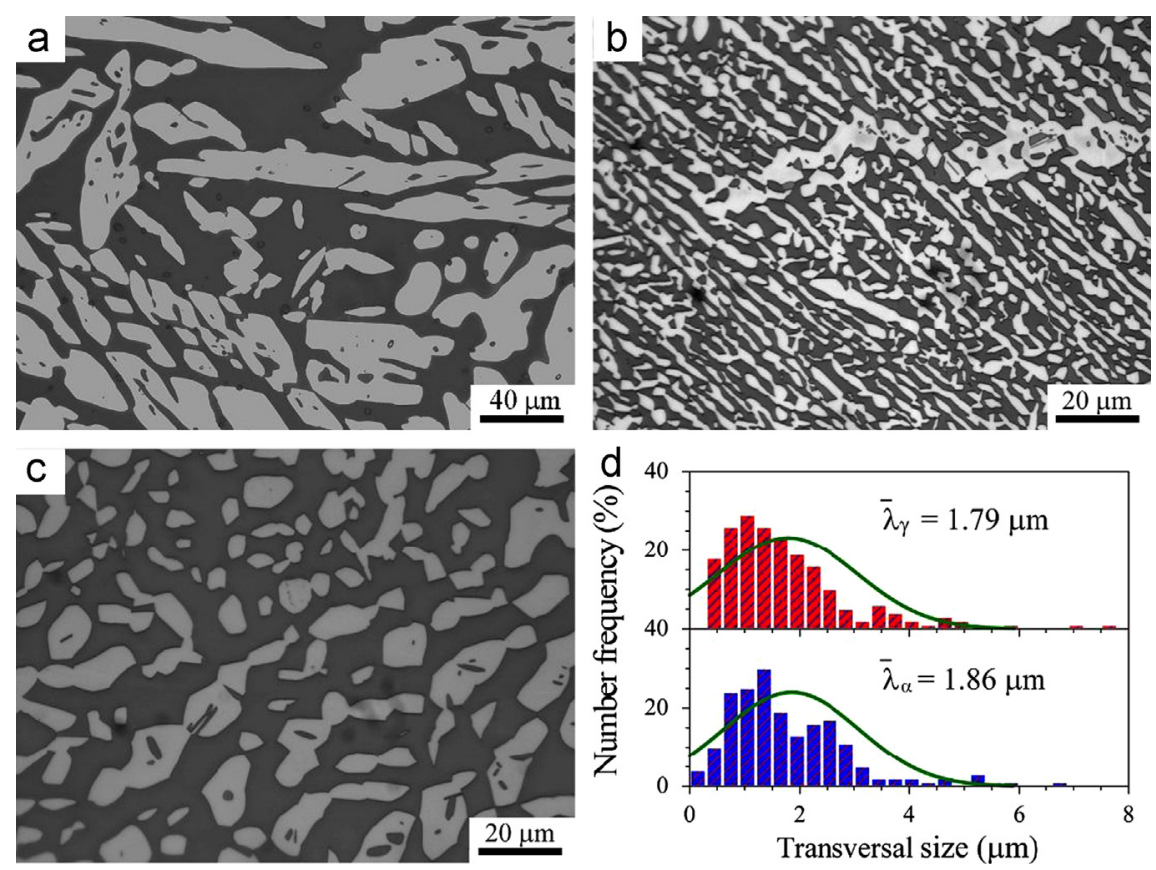

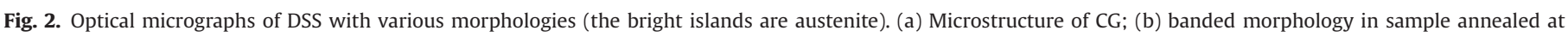

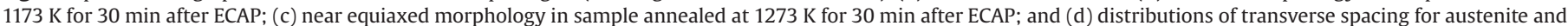
ferrite in (b). 

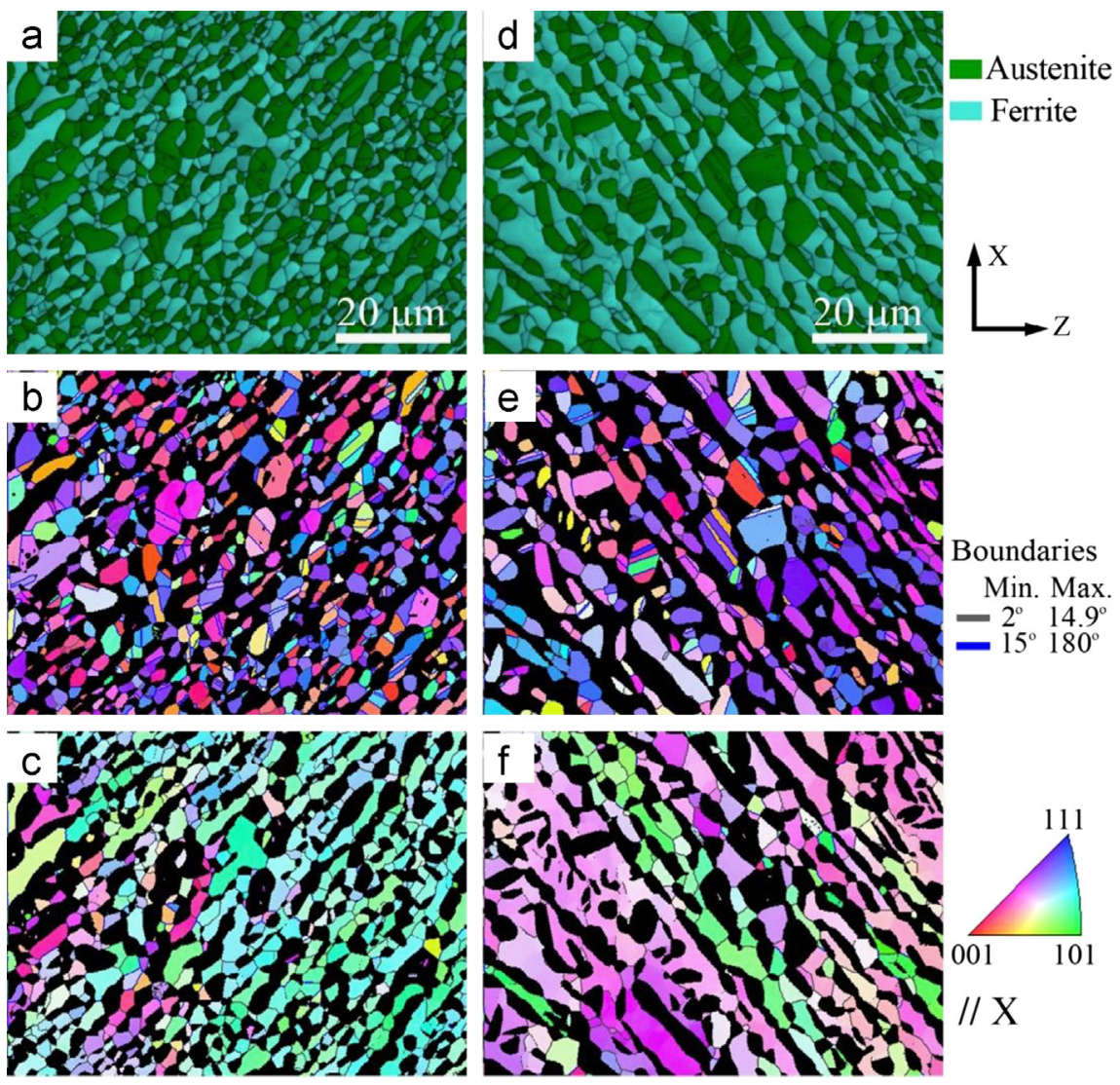

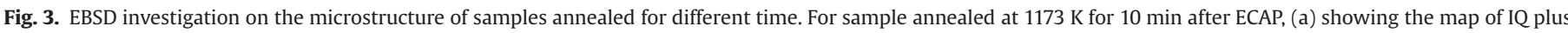

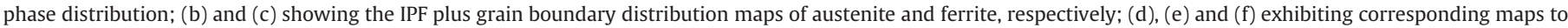
(a), (b) and (c) for sample annealed for $20 \mathrm{~min}$.
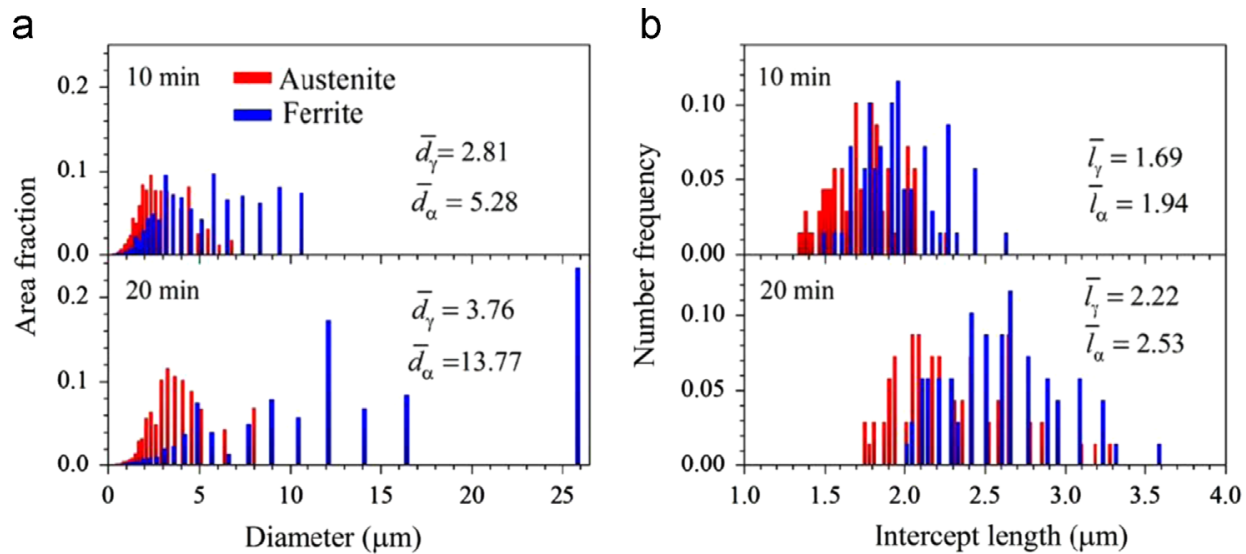

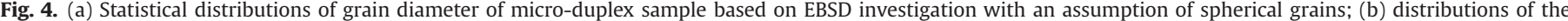
intercept length of grains.

found that the austenite of this DSS is relatively stable even during ECAP, and less than $5 \mathrm{vol} \%$ of austenite was transformed after 4 passes pressing at room temperature [30].

Inhomogeneous plastic strain distribution would occur during tensile deformation as the austenite and ferrite have different strengths due to their different yield stresses and strain hardening rates. Thus plastic strain gradient arises across phase boundary and GNDs are generated to meet the requirement of strain gradient. The kernel average misorientation (KAM) map, calculated from local orientation gradients, is used to reflect the GNDs density. Fig. 6(a) and (b) displays the KAM maps of austenite experienced at 0 and 0.2 tensile strains respectively, and the corresponding maps of ferrite are shown in Fig. 6(c) and (d). Before tensile deformation, the KAM maps of Fig. 6(a) and (c) indicate that the some GNDs have existed in both austenite and ferrite. This is probably produced by plastic deformation at oil quenching stage due to different thermal expansion coefficients between two phases. After 0.2 tensile strain, the KAM maps of Fig. 6(b) and (d) display higher values, indicating the generation of new GNDs during tensile deformation.

The change of KAM values during tensile test is further expressed by their statistical distributions. As shown in Fig. 7, the peak positions of austenite and ferrite move right after 
0.2 tensile straining, clearly manifesting the formation of GNDs. In addition, the austenite has a slightly higher peak value than that of the ferrite, indicating a little more storage of GNDs in austenite.

\section{Discussion}

\subsection{Strengthening mechanism of dual phase microstructure}

In micro-duplex structure, a large number of phase boundaries were introduced, and thus the mechanical properties may largely depend on the phase boundaries rather than grain boundaries. It is therefore necessary to investigate the effects of phase interaction on the mechanical behaviors of dual phase materials. Phase

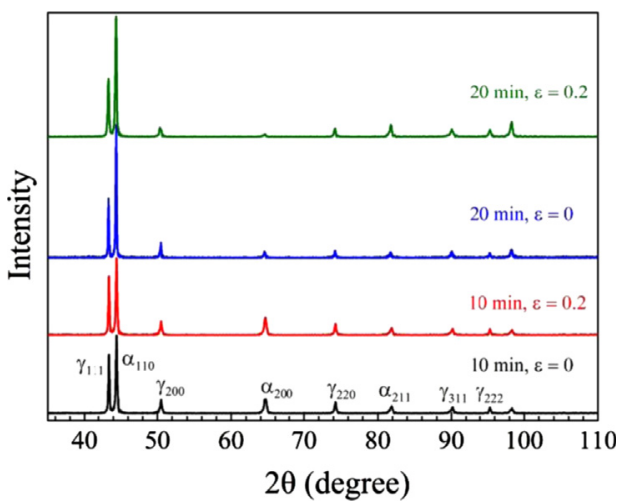

Fig. 5. XRD spectra of two micro-duplex samples before and after tensile deformation boundary could be a strong barrier to obstruct the dislocation motion [25]. Considering the Hall-Petch type relationship, the strengthening coefficient of phase boundary in DSS is measured quantitatively, and then the contributions of phase and grain boundaries on the overall strength are separated.

The dependence of yield stress on grain size for individual phase could be expressed by the classic Hall-Petch relations:

$\sigma_{y \gamma}=\sigma_{0 \gamma}+k_{\gamma} d_{\gamma}^{-1 / 2}$

$\sigma_{y \alpha}=\sigma_{0 \alpha}+k_{\alpha} d_{\alpha}^{-1 / 2}$

The first terms on the right hands of Eqs. (1a) and (1b) represent the contribution of lattice friction and solid solution of chemical elements. Fan et al. [36,37] had extended the Hall-Petch

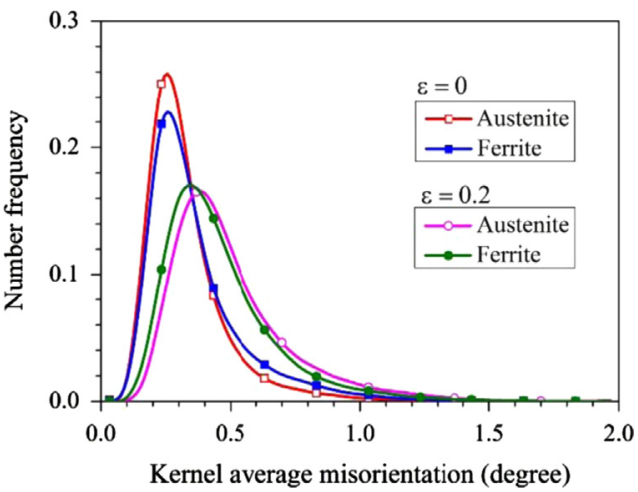

Fig. 7. Distributions of KAM values for austenite and ferrite before and after tensile deformation in sample annealed for $10 \mathrm{~min}$.
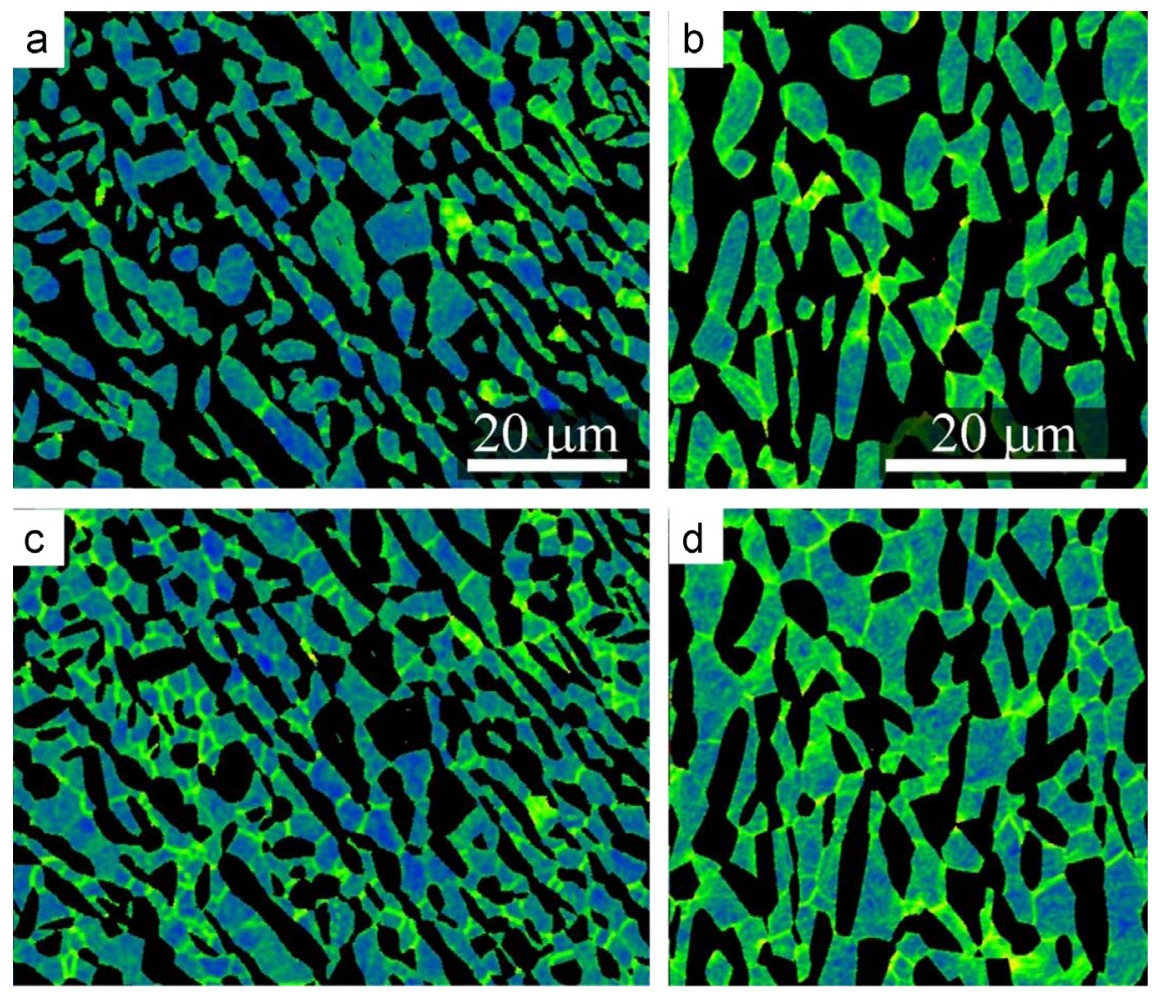

$\underset{0^{\circ}}{\operatorname{Min}} \underset{2^{\circ}}{\operatorname{Max}}$

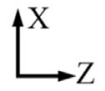

Fig. 6. Compatible deformation of micro-duplex sample annealed for 10-min. (a) KAM maps of austenite before tensile deformation; (b) KAM maps of austenite after 0.2 tensile strain; (c) and (d) showing the corresponding maps of ferrite to (a) and (b). 
relationship to describe the size dependence of yield stress of dual phase materials. Within this method, as shown in Fig. 8, the dual phase microstructure with any grain size, grain shape and phase distribution could be transformed into an idealized microstructure, which consists of two single phase parts subdivided by only grain boundaries and one dual phase part including phase boundaries alone. Therefore, the yield stress of dual phase microstructure could be expressed as

$\sigma_{\mathrm{yC}}=\left(\sigma_{0 \gamma}+k_{\gamma} d_{\gamma}^{-1 / 2}\right) \cdot q_{\gamma}+\left(\sigma_{0 \alpha}+k_{\alpha} d_{\alpha}^{-1 / 2}\right) \cdot q_{\alpha}+\left(\sigma_{0 \alpha \gamma}+k_{\alpha \gamma} \bar{d}_{\alpha \gamma}^{-1 / 2}\right) \cdot q_{\alpha \gamma}$,

where $q_{\gamma}, q_{\alpha}$ and $q_{\alpha \gamma}$ are the volume fractions of $\gamma, \alpha$ and $\alpha-\gamma$ parts in the idealized microstructure respectively; $\bar{d}_{\alpha \gamma}$ is a volumefraction-weighted grain size in $\alpha-\gamma$ part. In order to calculate $q_{\gamma}$, $q_{\alpha}, q_{\alpha \gamma}$ and $\bar{d}_{\alpha \gamma}$, two parameters of contiguities $\left(C_{\gamma}\right.$ and $\left.C_{\alpha}\right)$ and separations $\left(S_{\gamma}\right.$ and $\left.S_{\alpha}\right)$ are introduced and can be measured experimentally [36,37].

$C_{\gamma}=\frac{2 N_{\mathrm{L}}^{\gamma \gamma}}{2 N_{\mathrm{L}}^{\gamma \gamma}+N_{\mathrm{L}}^{\alpha \gamma}}$

$C_{\alpha}=\frac{2 N_{\mathrm{L}}^{\alpha \alpha}}{2 N_{\mathrm{L}}^{\alpha \alpha}+N_{\mathrm{L}}^{\alpha \gamma}}$

$S_{\gamma}=\frac{N_{\mathrm{L}}^{\alpha \gamma}}{2 N_{\mathrm{L}}^{\gamma \gamma}+N_{\mathrm{L}}^{\alpha \gamma}}$

$S_{\alpha}=\frac{N_{\mathrm{L}}^{\alpha \gamma}}{2 N_{\mathrm{L}}^{\alpha \alpha}+N_{\mathrm{L}}^{\alpha \gamma}}$

where $N_{\mathrm{L}}^{\gamma \gamma}, N_{\mathrm{L}}^{\alpha \alpha}$ and $N_{\mathrm{L}}^{\alpha \gamma}$ are the intercept numbers of $\gamma / \gamma, \alpha / \alpha$ and $\alpha / \gamma$ interfaces with a random line of unit length on a polished plane. Then, $q_{\gamma}, q_{\alpha}, q_{\alpha \gamma}$ and $\bar{d}_{\alpha \gamma}$ in Eq. (2) could be calculated by

$q_{\gamma}=C_{\gamma} f_{\gamma}$,

$q_{\alpha}=C_{\alpha} f_{\alpha}$

$q_{\alpha \gamma}=S_{\gamma} f_{\gamma}+S_{\alpha} f_{\alpha}$

$\bar{d}_{\alpha \gamma}=\frac{S_{\gamma} f_{\gamma} d_{\gamma}+S_{\alpha} f_{\alpha} d_{\alpha}}{q_{\alpha \gamma}}$

where $f_{\gamma}$ and $f_{\alpha}$ are the volume fractions of $\gamma$ and $\alpha$ phases. The yield stress of dual phase microstructure can also be expressed as

$\sigma_{\mathrm{yC}}=\sigma_{0 \mathrm{C}}+k_{\mathrm{C}} \bar{d}^{-1 / 2}$

where $\bar{d}\left(=d_{\gamma} f_{\gamma}+d_{\alpha} f_{\alpha}\right)$ is the average grain size; $\sigma_{0 \mathrm{C}}$ and $k_{\mathrm{C}}$ are the

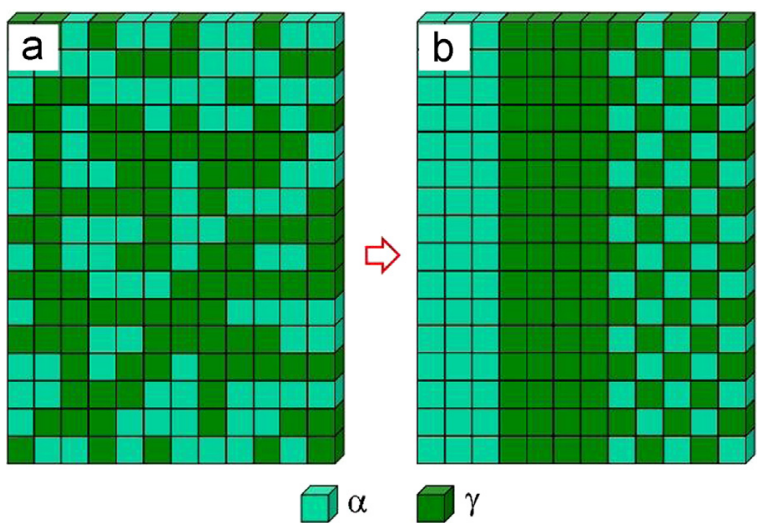

Fig. 8. Schematic illustration of the topological transformation [36]. (a) The dual phase microstructure with randomly distributed phases and (b) the transformed body consisting of three regular parts. overall friction stress and Hall-Petch coefficient respectively, which could be determined experimentally. Based on Eqs. (2) and (5), $\sigma_{0 \alpha \gamma}$ could be derived as

$\sigma_{0 \alpha \gamma}=\frac{\sigma_{0 C}-q_{\gamma} \sigma_{0 \gamma}-q_{\alpha} \sigma_{0 \alpha}}{q_{\alpha \gamma}}$

Some of the microstructural parameters, such as $d_{\gamma}, d_{\alpha}, \bar{d}_{\alpha \gamma}, q_{\gamma}$, $q_{\alpha}$ and $q_{\alpha \gamma}$, could be experimentally determined and mathematically calculated by Eq. (4). The intercepts $\left(\sigma_{0 \gamma}, \sigma_{0 \alpha}\right)$ and slope parameters $\left(k_{\gamma}, k_{\alpha}\right)$ were expected to be obtained from the samples with single $\gamma$ and $\alpha$ phases, respectively. However, it is difficult to fabricate a single phase alloy which possesses the same chemical compositions as those in the dual phase materials. Hirota et al. [38] measured the $\sigma_{0 \gamma}$ and $\sigma_{0 \alpha}$ of another DSS using microhardness testing. Here we show a simple method to obtain $\sigma_{0 \gamma}, \sigma_{0 \alpha}$, $k_{\gamma}$ and $k_{\alpha}$ by Pickerinǵs equation [39] and multiple linear regression analysis.

The alloying elements have strong effects on the intercepts of both phases. Sieurin et al. [40] had deduced the hardening coefficients of chemical elements by linear regression, which was shown as $\sigma_{0 \gamma}(\mathrm{MPa})=700 f_{\mathrm{Cr}}+2000 f_{\mathrm{Mn}}+3300 f_{\mathrm{Si}}+290 f_{\mathrm{Ni}}+$ $770 \sqrt{f_{N}}$, where the symbols $f$ denote the weight fractions of chemical elements (e.g., $f_{\mathrm{Cr}}=\mathrm{wt}$. $\mathrm{Cr}_{\mathrm{r}} / \mathrm{wt}$.total $)$, so they are dimensionless parameters. Therefore, all the coefficients on the right side of this equation are in units of MPa. Since the concentrations of alloying elements are not the same in the two phases $\alpha$ and $\gamma$, the real chemical compositions in two phases (as shown in Table 1) are obtained by considering the equilibrium distribution factors suggested by Charles [41]. Thus, the $\sigma_{0 \gamma}$ in UNS S32304 is calculated through multiplying the experimental $\sigma_{0 \gamma}$ of $\mathrm{Fe}-25 \mathrm{Cr}-$ $7 \mathrm{Ni}$ alloy by a ratio considering the difference in chemical concentration. The measured value of $\sigma_{0 \gamma}$ in $\mathrm{Fe}-25 \mathrm{Cr}-7 \mathrm{Ni}$ alloy is $345 \mathrm{MPa}$ [38]. The sum of contributions of all elements to $\sigma_{0 \gamma}$ is 283.7 MPa in $\mathrm{Fe}-25 \mathrm{Cr}-7 \mathrm{Ni}$ and 246.4 MPa in UNS S32304. Thus the ratio is equal to 246.4/283.7, and $\sigma_{0 \gamma}$ of UNS S32304 can be estimated as $(246.4 / 283.7) 345 \mathrm{MPa}=299.6 \mathrm{MPa}$.

The $\sigma_{0 \mathrm{C}}$ and $k_{\mathrm{C}}$ could be calculated through multiple linear regression by considering five different dual phase microstructures listed in Table 2, which gives $\sigma_{0 \mathrm{C}}=314.8 \mathrm{MPa}$ and $k_{\mathrm{C}}=$ 333.4 MPa $\mu m^{1 / 2}$. The overall friction stress of ferrite in DSS could not be derived as that in austenite because the function is not simply linear any more due to high concentration of $\mathrm{Cr}[42,43]$. Since $\sigma_{0 \mathrm{C}}$ could be expressed as $\sigma_{0 \mathrm{C}}=\sigma_{0 \gamma} f_{\gamma}+\sigma_{0 \alpha} f_{\alpha}$ and the volume fraction of austenite is about $0.45, \sigma_{0 \alpha}$ can be obtained as $326.9 \mathrm{MPa}$.

The friction stresses of austenite and ferrite have been obtained now, retaining only three unknown parameters in Eq. (2): $k_{\gamma}, k_{\alpha}$ and $k_{\alpha \gamma}$. However, there are five independent equations based on the data shown in Table 2 , and thus the $k_{\gamma}, k_{\alpha}$ and $k_{\alpha \gamma}$ could be calculated by multiple linear regression. The results are displayed in Table 3, from which the phase boundary appears a moderate coefficient (307.9 MPa $\mu \mathrm{m}^{1 / 2}$ ) as compared to those of grain boundaries of austenite $\left(224.9 \mathrm{MPa} \mu \mathrm{m}^{1 / 2}\right)$ and ferrite $\left(428.9 \mathrm{MPa} \mu \mathrm{m}^{1 / 2}\right)$. The coefficient of phase boundary is in agreement with the investigation of Fan el al. (345 MPa $\mu \mathrm{m}^{1 / 2}$ ) [36,44].

During plastic deformation, the dislocation transmission across phase boundary could occur in DSS when the slip planes in austenite and ferrite are nearly parallel [45]. It is the possible

Table 1

Chemical compositions (wt\%) of $\gamma$ - and $\alpha$-phase in UNS S32304, which is calculated using the equilibrium distribution factor investigated by Charles [41].

\begin{tabular}{|c|c|c|c|c|c|c|c|c|}
\hline & $C$ & $\mathrm{Si}$ & $\mathrm{Mn}$ & $\mathrm{Cr}$ & $\mathrm{Ni}$ & Mo & $\mathrm{N}$ & $\mathrm{P}$ \\
\hline$\gamma$ & 0.02 & 0.45 & 1.26 & 22.02 & 5.00 & 0.31 & 0.24 & 0.01 \\
\hline$\alpha$ & 0.02 & 0.55 & 1.14 & 24.93 & 3.00 & 0.49 & 0.02 & 0.03 \\
\hline
\end{tabular}


Table 2

Summary of the obtained yield stresses, volume fractions, average grain diameters and topological parameters.

\begin{tabular}{|c|c|c|c|c|c|c|c|c|c|c|}
\hline Sample & $\sigma_{\mathrm{yc}}(\mathrm{MPa})$ & $f_{\gamma}$ & $f_{\alpha}$ & $d_{\gamma}(\mu \mathrm{m})$ & $d_{\alpha}(\mu \mathrm{m})$ & $q_{\gamma}$ & $q_{\alpha}$ & $q_{\alpha \gamma}$ & $\bar{d}_{\alpha \gamma}(\mu \mathrm{m})$ & $\bar{d}(\mu \mathrm{m})$ \\
\hline \multirow[t]{2}{*}{ CG [48] } & 403 & $0.45 \pm 0.01$ & 0.55 & 17.6 & 19.5 & 0.13 & 0.13 & 0.74 & 18.68 & 18.65 \\
\hline & 457 & $0.39 \pm 0.01$ & 0.61 & 4.81 & 5.72 & 0.11 & 0.13 & 0.76 & 5.38 & 5.37 \\
\hline \multirow[t]{2}{*}{ Micro-duplex } & 540 & $0.48 \pm 0.03$ & 0.52 & 1.69 & 1.94 & 0.15 & 0.13 & 0.72 & 1.83 & 1.82 \\
\hline & 517 & $0.49 \pm 0.04$ & 0.51 & 2.22 & 2.53 & 0.18 & 0.18 & 0.64 & 2.38 & 2.38 \\
\hline NS [30] & 1100 & $0.45 \pm 0.02$ & 0.55 & 0.11 & 0.24 & 0.44 & 0.54 & 0.02 & 0.20 & 0.18 \\
\hline
\end{tabular}

Table 3

Hall-Petch coefficients of duplex stainless steel obtained by multiple linear regressions. The statistical parameters are $R^{2}=0.999$ and $p=10^{-3}$. (Unit: $\mathrm{MPa} \mu \mathrm{m}^{1 / 2}$ ).

\begin{tabular}{lllll}
\hline Alloys & $k_{\gamma}$ & $k_{\alpha}$ & $k_{\alpha \gamma}$ & Refs. \\
\hline UNS S32304 & 224.9 & 428.9 & 309.7 & This study \\
Fe-Cr-Ni & 458 & 281 & 345 & {$[36]$} \\
SUS 316L & 164 & - & - & {$[38]$} \\
Fe-25Cr-7Ni-0.15N & 239 & 954 & - & {$[38]$} \\
\hline
\end{tabular}

reason for the moderate coefficient of phase boundary. In addition, contrary to the investigation of Fan et al. [36] but in accordance with the result of Sieurin et al. [40], the ferrite in the present study has a larger coefficient than that of the austenite. Since the coefficient depends closely on the chemical compositions, the segregation of solute atoms at grain boundaries may raise the coefficient of ferrite.

As all the parameters in Eq. (2) have been obtained, the yield stresses of dual phase materials with different grain sizes can be calculated. Fig. 9 exhibits the calculated and experimental values of yield stress, and the result indicates a good agreement between them.

\subsection{Effect of phase interaction on strain hardening rate}

One characteristic for the plastic deformation of dual phase materials is the plastic strain gradient across phase boundaries induced by the inhomogeneous distribution of plastic strain. According to the plastic strain gradient theory, it requires the generation of GNDs to make the deformation compatible. There is no obvious difference in physics between the GNDs and traditional lattice dislocations, but the GNDs in essence are an extra storage of dislocations due to geometrical necessity. It is the reason why extra strength occurs when plastic strain gradient exists. Thus, the strain hardening rate of dual phase material would be influenced by this extra strength since the strain gradient exists all through the tensile deformation.

The gradient plastic strain in dual phase microstructure is schematically illustrated in Fig. 10. Assuming linear variation of plastic strain with distance, the absolute value of strain gradient in space therefore remains constant in dual phase part as shown in Fig. 8. Based on the strain gradient theory, the flow stress of dual phase material can be expressed as

$\sigma^{\prime}=q_{\gamma} \sigma_{\gamma}+q_{\alpha} \sigma_{\alpha}+q_{\alpha \gamma} \sigma_{\alpha \gamma}^{\prime}$,

where $\sigma_{\alpha \gamma}^{\prime}$ denotes the flow stress of dual phase part in Fig. 8, which has incorporated the effect of strain gradient. Taking into account the works of Nix and Gao [46], the $\sigma_{\alpha \gamma}^{\prime}$ could be expressed as

$\frac{\sigma_{\alpha \gamma}^{\prime}}{\sigma_{\alpha \gamma}}=\sqrt{1+l \chi}$

where $l$ is a characteristic material length scale, and $\chi$ is the strain gradient. Substituting Eq. (8) into Eq. (7), and incorporating a stress

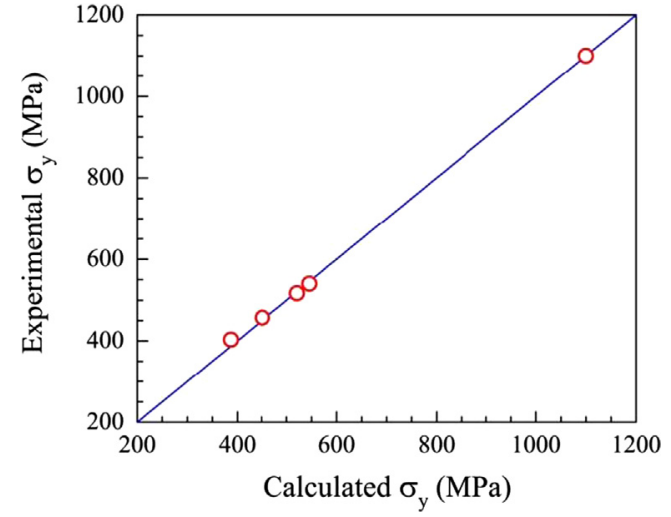

Fig. 9. Comparison of the yield stresses between theoretical predictions and experimental results for different DSS samples.

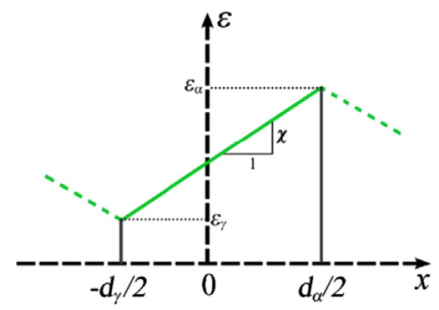

Fig. 10. Schematic illustration of the gradient plastic strain in dual phase microstructure. For simplicity, the plastic strain is varied linearly with distance, and therefore generates a constant absolute value of strain gradient in dual phase part.

partitioning coefficient $p_{\alpha \gamma}$, one can easily get

$\frac{\sigma^{\prime}}{\sigma}=1+q_{\alpha \gamma} p_{\alpha \gamma}\left\{[1+l \chi]^{1 / 2}-1\right\}$,

where $p_{\alpha \gamma}$ is equal to $\sigma_{\alpha \gamma} / \sigma$, and the characteristic material length scale is expressed as

$l=b\left(\frac{u}{q_{\alpha \gamma} \sigma}\right)^{2}$,

where $b$ is the magnitude of Burgers vector, and $u$ is the shear modulus. Based on Eq. (9), it is clear that the more the volume fraction of dual phase part and the larger the strain gradient are, the higher the strength becomes.

In order to analyze the strain hardening behavior, MeckingKocks theory was always used and exhibited a linear equation with respect to flow stress [47]:

$\Theta=\Theta_{0}-K\left(\sigma-\sigma_{0}\right)$,

where $\Theta=d \sigma / d \varepsilon, \Theta_{0}$ is an athermal hardening rate, and $K$ denotes the rate of dynamic recovery. Taking derivatives of Eqs. (9) and (10) with respect to strain, combining the differential equations, and assuming nearly constant $p_{\alpha \gamma}$, the strain hardening rate of dual phase material which incorporates the strain gradient 
Table 4

Summary of surface-to-volume ratios $\left(s_{\mathrm{V}}\right)$ of phase boundary in different microstructures, as well as the experimental (Exp.) and theoretical (Theor.) values of dynamic recovery rate.

\begin{tabular}{lllll}
\hline Sample & $\begin{array}{l}S_{\mathrm{V}} \\
\left(\times 10^{5} \mathrm{~m}^{-1}\right)\end{array}$ & $\begin{array}{l}\Theta_{0}^{\prime} \\
(\mathrm{MPa})\end{array}$ & $\begin{array}{l}K\left(1-\zeta S_{\mathrm{V}}\right) \\
(\text { Exp. })\end{array}$ & $\begin{array}{l}K\left(1-\zeta s_{\mathrm{V}}\right) \\
(\mathrm{Theor})\end{array}$ \\
\hline CG & 1.2 & $2802.7 \pm 26.1$ & $4.31 \pm 0.4$ & 4.11 \\
Micro-duplex & 11.7 & $2468.3 \pm 76.7$ & $2.80 \pm 0.6$ & 2.76 \\
& 8.1 & $2685.4 \pm 87.2$ & $3.44 \pm 0.9$ & 3.21 \\
\hline
\end{tabular}

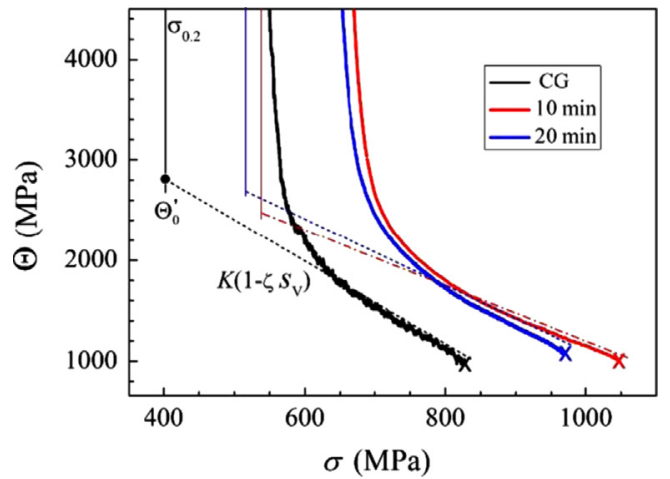

Fig. 11. The strain hardening rate $v s$. true stress curves upon tensile deformation of CG and micro-duplex samples.

effect was obtained (detailed derivation process is shown in Appendix A section):

$\Theta^{\prime}=\Theta_{0}^{\prime}-K\left(1-\zeta s_{V}\right)\left(\sigma-\sigma_{0}\right)$,

where $s_{\mathrm{V}}$ is the surface-to-volume ratio of phase boundary, equaling to $3 q_{\alpha \gamma} / \bar{d}_{\alpha \gamma}$ when the grains in dual phase part are considered as spherical.

Based on Eq. (12), the dynamic recovery rate of dual phase material depends closely on the surface-to-volume ratio of phase boundary. The higher the ratio is, the lower the dynamic recovery rate becomes, and vice versa.

The values of $s_{\mathrm{V}}, K\left(1-\zeta s_{\mathrm{V}}\right)$ and $\Theta_{0}^{\prime}$ are listed in Table $4 . K$ and $\zeta$ are obtained as 4.26 and $0.03 \mathrm{~m}$ by the linear regression method respectively. Therefore, the theoretical $K\left(1-\zeta s_{\mathrm{V}}\right)$ of three different duplex structures can be calculated, and the results demonstrated that the calculated $K\left(1-\zeta s_{\mathrm{V}}\right)$ is in good agreement with experimental results.

Fig. 11 show the comparison of theoretical $\Theta-\sigma$ data with those obtained from experiments at linear decreasing stage. The results show that theoretical predictions have only little overestimations on the dynamic recovery rate. In general, the model as expressed by Eq. (12) could well describe the influence of phase interaction on strain hardening behavior of duplex stainless steel.

\section{Conclusion}

Micro-duplex structured samples consisting of austenite and ferrite (with average grain sizes near $2 \mu \mathrm{m}$ in both phases) were fabricated by ECAP and subsequent thermal annealing. As compared to their coarse-grained counterpart, the micro-duplex structures have finer grain sizes in both phases and higher surface-to-volume ratios of phase boundary. The yield stress and uniform elongation of micro-duplex samples are increased simultaneously. The strengthening mechanism is quantitatively investigated, and the influence of surface-to-volume ratio of phase boundary on strain hardening behavior is analyzed based on strain gradient theory. The main conclusions are summarized as follows:

1. The average grain sizes of austenite and ferrite are 1.69 and $1.94 \mu \mathrm{m}$ in micro-duplex structure annealed at $1173 \mathrm{~K}$ for 10 min respectively, while the yield stress and uniform elongation increase to $540 \mathrm{MPa}$ and 0.3 as compared to $403 \mathrm{MPa}$ and 0.26 of their coarse-grained counterpart respectively.

2. The $\alpha / \gamma$ interface has an intermediate ability to impede the motion of dislocations as compared to $\gamma / \gamma$ and $\alpha / \alpha$ grain boundaries. A Hall-Petch type coefficient is used to quantitatively describe this strengthening ability, which are measured as 309.7, 224.9 and $428.9 \mathrm{MPa} \mu \mathrm{m}^{1 / 2}$ for $\alpha / \gamma, \gamma / \gamma$ and $\alpha / \alpha$ interfaces, respectively.

3. The surface-to-volume ratio of phase boundary in microduplex structure is estimated to be $1.17 \times 10^{6} \mathrm{~m}^{-1}$, which is increased by an order of magnitude as compared to $1.2 \times 10^{5} \mathrm{~m}^{-1}$ of its coarse-grained counterpart.

4. The tensile test shows that the dynamic recovery rate is decreased significantly in micro-duplex structure. Based on strain gradient theory, a simple model was proposed to describe the influence of surface-to-volume ratio of phase boundary on strain hardening rate, which shows good agreement with experimental results.

\section{Acknowledgments}

The authors would like to thank the financial supports by 973 Program of China (Nos. 2012CB932203 and 2012CB937500) and NSFC (Grants nos. 51301187, 11222224, 11021262 and 11072243).

\section{Appendix A}

Since the flow stress of dual phase microstructure incorporating the effect of plastic strain gradient has been expressed as Eq. (9), the strain hardening rate can be obtained by taking the derivative of both sides with respect to strain:

$\frac{d \sigma^{\prime}}{d \varepsilon}=\left\{1+q_{\alpha \gamma} p_{\alpha \gamma}\left[(1+l \chi)^{1 / 2}-1\right]\right\} \cdot \frac{d \sigma}{d \varepsilon}+\frac{q_{\alpha \gamma} p_{\alpha \gamma}}{2(1+l \chi)^{1 / 2}}\left(\chi \frac{d l}{d \varepsilon}+l \frac{d \chi}{d \varepsilon}\right) \cdot \sigma$

In the equation above, the characteristic material length scale $l$ and strain gradient $\chi$ are strain dependent. The variation of $p_{\alpha \gamma}$ with plastic strain was neglected for simplicity. Taking Eq. (10) into account, the derivative of $l$ over $\varepsilon$ is equal to

$\frac{d l}{d \varepsilon}=-\frac{2 l}{p_{\alpha \gamma}^{2}} \frac{1}{\sigma} \frac{d \sigma}{d \varepsilon}$

Substituting Eq. (A2) into Eq. (A1), Eq. (A1) can be rewritten as

$$
\begin{aligned}
\frac{d \sigma^{\prime}}{d \varepsilon}= & \left\{1+q_{\alpha \gamma} p_{\alpha \gamma}\left[(1+l \chi)^{1 / 2}-1\right]-\frac{q_{\alpha \gamma}}{p_{\alpha \gamma}} \frac{l \chi}{(1+l \chi)^{1 / 2}}\right\} \cdot \frac{d \sigma}{d \varepsilon} \\
& +\frac{l q_{\alpha \gamma}}{2 p_{\alpha \gamma}(1+l \chi)^{1 / 2}} \frac{d \chi}{d \varepsilon} \cdot \sigma
\end{aligned}
$$

Substituting Eq. (11) into Eq. (A3), one can get

$$
\frac{d \sigma^{\prime}}{d \varepsilon}=\left(\Theta_{0}+K \sigma_{0}\right)\left\{1-q_{\alpha \gamma} p_{\alpha \gamma}\left[1+\frac{l \chi\left(1-p_{\alpha \gamma}^{2}\right)-p_{\alpha \gamma}^{2}}{(1+l \chi)^{1 / 2} p_{\alpha \gamma}^{2}}\right]\right\}
$$




$$
-\left\{K\left\{1-q_{\alpha \gamma} p_{\alpha \gamma}\left[1+\frac{l \chi\left(1-p_{\alpha \gamma}^{2}\right)-p_{\alpha \gamma}^{2}}{(1+l \chi)^{1 / 2} p_{\alpha \gamma}^{2}}\right]\right\}-\frac{q_{\alpha \gamma}}{2 p_{\alpha \gamma}} \frac{l}{(1+l \chi)^{1 / 2}} \frac{d \chi}{d \varepsilon}\right\} \cdot \sigma
$$

If the difference of plastic strain between two phases is relatively constant, Eq. (A4) can be simplified as

$$
\begin{aligned}
\frac{d \sigma^{\prime}}{d \varepsilon}= & \underbrace{\Theta_{0}\left\{1-q_{\alpha \gamma} p_{\alpha \gamma}\left[1+\frac{l \chi\left(1-p_{\alpha \gamma}^{2}\right)-p_{\alpha \gamma}^{2}}{(1+l \chi)^{1 / 2} p_{\alpha \gamma}^{2}}\right]\right\}}_{\Theta_{0}^{\prime}} \\
& -\underbrace{K\left\{1-q_{\alpha \gamma} p_{\alpha \gamma}\left[1+\frac{l \chi\left(1-p_{\alpha \gamma}^{2}\right)-p_{\alpha \gamma}^{2}}{(1+l \chi)^{1 / 2} p_{\alpha \gamma}^{2}}\right]\right\}}_{K^{\prime}} \cdot\left(\sigma-\sigma_{0}\right)
\end{aligned}
$$

Therefore, Eqs. (A5) and (11) are identical in form. Since the surface-to-volume ratio $s_{\mathrm{V}}$ of phase boundary is equal to $3 q_{\alpha \gamma} / \bar{d}_{\alpha \gamma}$ when the grains are assumed spherical in dual phase part, Eq. (A5) can be further rewritten as

$\frac{d \sigma^{\prime}}{d \varepsilon}=\Theta_{0}^{\prime}-K\left(1-\zeta s_{V}\right)\left(\sigma-\sigma_{0}\right)$

where $\zeta$ is equal to $\bar{d}_{\alpha \gamma} / 3\left[1+\left(\left(l \chi\left(1-p_{\alpha \gamma}^{2}\right)-p_{\alpha \gamma}^{2}\right) /(1+l \chi)^{1 / 2} p_{\alpha \gamma}^{2}\right)\right]$; $\Theta_{0}^{\prime}$ is equal to $\Theta_{0}\left(1-\zeta s_{\mathrm{V}}\right)$.

\section{References}

[1] T.H. Fang, W.L. Li, N.R. Tao, K. Lu, Science 331 (2011) 1587-1590.

[2] K. Lu, L. Lu, S. Suresh, Science 324 (2009) 349-352.

[3] Y.M. Wang, E. Ma, Acta Mater. 52 (2004) 1699-1709.

[4] Y.T. Zhu, X.Z. Liao, Nat. Mater. 3 (2004) 351-352.

[5] Y.M. Wang, E. Ma, M.W. Chen, Appl. Phys. Lett. 80 (2002) 2395-2397.

[6] X.L. Wu, P. Jiang, L. Chen, F.P. Yuan, Y.T.T. Zhu, Proc. Natl. Acad. Sci. 111 (2014) 7197-7201.

[7] Y.M. Wang, M.W. Chen, F.H. Zhou, E. Ma, Nature 419 (2002) 912-915.

[8] Y.H. Zhao, X.Z. Liao, S. Cheng, E. Ma, Y.T. Zhu, Adv. Mater. 18 (2006) 2280-2283.

[9] G. Liu, G.J. Zhang, F. Jiang, X.D. Ding, Y.J. Sun, J. Sun, E. Ma, Nat. Mater. 12 (2013) 344-350.

[10] X. Wu, N. Tao, Y. Hong, J. Lu, K. Lu, Scr. Mater. 52 (2005) 547-551.

[11] Y.Q. Ma, J.E. Jin, Y.K. Lee, Scr. Mater. 52 (2005) 1311-1315.

[12] C.C. Koch, R.O. Scattergood, K.L. Murty, JOM 59 (2007) 66-70.

[13] M. Militzer, Science 298 (2002) 975-976.
[14] N. Jia, R. Lin Peng, Y.D. Wang, S. Johansson, P.K. Liaw, Acta Mater. 56 (2008) $782-793$.

[15] S. Harjo, Y. Tomota, P. Lukáš, D. Neov, M. Vrána, P. Mikula, M. Ono, Acta Mater. 49 (2001) 2471-2479.

[16] R. Dakhlaoui, C. Braham, A. Baczmański, Mater. Sci. Eng. A 444 (2007) 6-17.

[17] N. Jia, R. Lin Peng, G.C. Chai, S. Johansson, Y.D. Wang, Mater. Sci. Eng. A 491 (2008) 425-433.

[18] W. Woo, V.T. Em, E.Y. Kim, S.H. Han, Y.S. Han, S.H. Choi, Acta Mater. 60 (2012) $6972-6981$.

[19] J. Wang, R.G. Hoagland, J.P. Hirth, A. Misra, Acta Mater. 56 (2008) 5685-5693.

[20] J. Wang, R.G. Hoagland, X.Y. Liu, A. Misra, Acta Mater. 59 (2011) 3164-3173.

[21] J. Wang, A. Misra, R.G. Hoagland, J.P. Hirth, Acta Mater. 60 (2012) 1503-1513.

[22] C.W. Sinclair, J.D. Embury, G.C. Weatherly, Mater. Sci. Eng. A 272 (1999) 90-98.

[23] L. He, L.F. Allard, E. Ma, Scr. Mater. 42 (2000) 517-523.

[24] S. Ankem, H. Margolin, Metall. Trans. A 14 (1983) 500-503.

[25] S. Ankem, H. Margolin, C.A. Greene, B.W. Neuberger, P.G. Oberson, Prog. Mater. Sci. 51 (2006) 632-709.

[26] D.H. Shin, K.T. Park, Mater. Sci. Eng. A 410 (2005) 299-302.

[27] Y.I. Son, Y.K. Lee, K.-T. Park, C.S. Lee, D.H. Shin, Acta Mater. 53 (2005) 3125-3134.

[28] R. Song, D. Ponge, D. Raabe, J.G. Speer, D.K. Madock, Mater. Sci. Eng. A 441 (2006) 1-17.

[29] Y.Z. Tian, S.D. Wu, Z.F. Zhang, R.B. Figueiredo, N. Gao, T.G. Langdon, Acta Mater. 59 (2011) 2783-2796.

[30] L. Chen, F.P. Yuan, P. Jiang, X.L. Wu, Mater. Sci. Eng. A 551 (2012) 154-159.

[31] T.G. Langdon, M. Furukawa, M. Nemoto, Z. Horita, JOM 52 (2000) 30-33.

[32] R.Z. Valiev, R.K. Islamgaliev, I.V. Alexandrov, Prog. Mater. Sci. 45 (2000) 103-189.

[33] Y. Iwahashi, Z. Horita, M. Nemoto, T.G. Langdon, Acta Mater. 46 (1998) 3317-3331.

[34] H. Kleemola, M. Nieminen, Metall. Trans. 5 (1974) 1863-1866.

[35] T. Maki, T. Furuhara, K. Tsuzaki, ISIJ Int. 41 (2001) 571-579.

[36] Z. Fan, P. Tsakiropoulos, P. Smith, A. Miodownik, Philos. Mag. A 67 (1993) $515-531$.

[37] Z. Fan, P. Tsakiropoulos, A.P. Miodownik, Mater. Sci. Technol. 8 (1992) 922-929.

[38] N. Hirota, F.X. Yin, T. Azuma, T. Inoue, Sci. Technol. Adv. Mater. 11 (2010) 025004.

[39] F.B. Pickering, R.W. Cahn, Constitution and Properties of Steels, VCH Verlasgesellschaft GmbH, Weinheim, 1992.

[40] H. Sieurin, J. Zander, R. Sandstrom, Mater. Sci. Eng. A 415 (2006) 66-71.

[41] J. Charles, in: T.G. Gooch (Ed.), Duplex Stainless Steels '94, The welding institute, Glasgow, 1994.

[42] I. Gutierrez, M.A. Altuna, Acta Mater. 56 (2008) 4682-4690.

[43] R. Rodriguez, I. Gutiérrez, Mater. Sci. Forum 426 (2003) 4525-4530.

[44] Y. Tomota, K. Kuroki, T. Mori, I. Tamura, Mater. Sci. Eng. 24 (1976) 85-94.

[45] B. Verhaeghe, F. Louchet, B. Doisneau-Cottignies, Y. Bréchet, J. Massoud, Philos. Mag. A 76 (1997) 1079-1091.

[46] W.D. Nix, H.J. Gao, J. Mech. Phys. Solids 46 (1998) 411-425.

[47] U.F. Kocks, H. Mecking, Prog. Mater. Sci. 48 (2003) 171-273.

[48] L. Chen, X.L. Wu, Mater. Sci. Forum 682 (2011) 123-130. 